Philosophie ANTIQUE

\section{Philosophie antique}

Problèmes, Renaissances, Usages

19 | 2019

L'épicurisme antique

\title{
Enrico PIERGIACOMI, Storia delle antiche teologie atomiste
}

Roma, Sapienza Università Editrice, 2017 (Collana Studi e Ricerche, 64), 431 p., ISBN 978-88-9377-040-8

\section{Giulia Scalas}

\section{(2) OpenEdition}

1 Journals

Édition électronique

URL : https://journals.openedition.org/philosant/1966

DOI : 10.4000/philosant. 1966

ISSN : 2648-2789

Éditeur

Éditions Vrin

\section{Édition imprimée}

Date de publication : 31 octobre 2019

Pagination : 196-198

ISBN : 978-2-7574-2534-3

ISSN : 1634-4561

Référence électronique

Giulia Scalas, «Enrico piergiacomı, Storia delle antiche teologie atomiste», Philosophie antique [En ligne], 19 | 2019, mis en ligne le 13 mai 2019, consulté le 02 décembre 2022. URL : http://

journals.openedition.org/philosant/1966; DOI : https://doi.org/10.4000/philosant.1966

\section{(ब) $\odot \Theta$}

Creative Commons - Attribution - Pas d'Utilisation Commerciale - Pas de Modification 4.0 International - CC BY-NC-ND 4.0

https://creativecommons.org/licenses/by-nc-nd/4.0/ 
Enrico Piergiacomi, Storia delle antiche teologie atomiste, Roma, Sapienza Università Editrice, 2017 (Collana Studi e Ricerche, 64), 431 p., ISBN 978-889377-040-8.

Cet ouvrage, Storia delle antiche teologie atomiste, est le fruit de la réélaboration d'une thèse de doctorat en philosophie rédigée et soutenue en 2016 par son auteur, Enrico Piergiacomi, à l'Université de Trente. L'objectif principal de ce travail transparaît déjà dans son titre : il s'agit, en effet, de chercher si et en quels termes il est légitime de parler de «théologies atomistes », et d'en reconstituer l'histoire. Cette étude vise à démontrer non seulement que la réflexion atomiste sur le divin est suffisamment solide et cohérente pour qu'on puisse la considérer comme une « théologie », donc comme un discours rationnel sur dieu, mais aussi qu'elle implique une représentation du divin qui joue un rôle important du point de vue éthique. L'ouvrage se construit ainsi en visant cette double cible, à la fois théorique et pratique.

Les quatre premiers chapitres visent à reconstituer les théories atomistes du divin de Démocrite (I), d'Épicure (II) et des épicuriens postérieurs, de Métrodore à Antiphane (III), et de Démétrius Lacon aux épicuriens d'époque impériale (IV). Le chapitre V porte sur les implications éthiques de ces théories. Dans le chapitre premier, l'auteur reconstitue la théologie de Démocrite - à partir, principalement, du fragment $\mathrm{B} 175 \mathrm{DK}$ et des témoignages de Sextus Empiricus (A 75 et B 166 DK) et de Plutarque (A 77 DK) en la confrontant à la conception homérique de la divinité. Le résultat est une théologie fondée sur l'idée d'un dieu anthropomorphe, comme celui d'Homère, dont l'existence

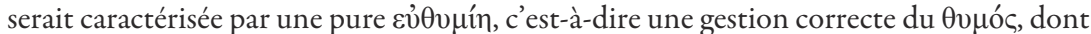
la bienveillance envers les hommes justes serait la conséquence. Malgré cette proximité avec le modèle homérique, le dieu de Démocrite est mortel, puisqu'il s'agit d'un composé et qu'aucun composé n'est indissoluble. En outre, il est étranger à la création de l'univers, puisque la cosmogonie est expliquée par le philosophe uniquement à partir du vortex atomique. L'explication démocritéenne du divin n'entre donc jamais en opposition avec les principes atomistes. Dans le deuxième chapitre, l'auteur reconstitue la théologie d'Épicure en s'appuyant en particulier sur les Lettres à Hérodote et à Ménécée et sur le De natura deorum de Cicéron. Ce qui frappe dans la méthodologie del'auteur est la volonté de faire émerger le plus rigoureusement possible la pensée même d'Épicure. Cette intention se manifeste clairement dans son choix de ne pas utiliser les témoignages, beaucoup plus nombreux, des épicuriens postérieurs pour reconstituer rétrospectivement la pensée du maitre, et de ne pas considérer a priori la théologie épicurienne comme unique et invariable tout au long de l'histoire du Jardin épicurien. D'autre part, cette intention est aussi perceptible dans le traitement et l'analyse de témoignages complexes comme ceux de Cicéron qui constituent à la fois un précieux réservoir d’informations pour la reconstitution de la théologie épicurienne, mais aussi un exemple de la réélaboration critique cicéronienne à laquelle fut souvent sujette la doctrine du Jardin. Le résultat de l'examen des textes ainsi mené est une conception soustractive du divin. Selon l'auteur, en effet, le dieu d'Épicure est hypo-humain. Pour le dire autrement, l'idée épicurienne du dieu ne serait que le résultat de la soustraction de tout ce qui est cause de malheur et de dissolution chez l'être humain. Le dieu serait donc un être anthropomorphe doué

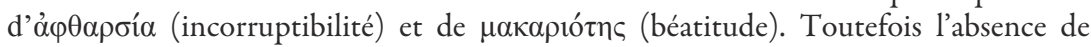
corruptibilité et de soucis ne sont pas les seuls « manques divins » qui caractérisent le dieu épicurien. En effet, l'auteur affirme que, selon Épicure, les dieux sont étrangers à toute forme d'activité. La théorie interprétative d'Enrico Piergiacomi dépend de ce qu'il appelle « l'antropologia epicurea ». En effet, à partir des textes épicuriens - les Sentences 


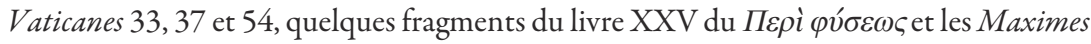
Capitales 20-21 -, l'auteur établit que la divinité épicurienne est privée du moteur de l'action, à savoir du désir. Puisque l'être humain agit toujours sous l'impulsion d'un désir, d'un manque qui provoque chez lui une souffrance, et que l'incorruptibilité, autrement dit l'absence de tout type de souffrance et de désir, caractérise la divinité, les dieux ne peuvent agir. Or, cet aspect de la théologie épicurienne semble se transformer dans le Jardin après la mort d'Épicure. En effet, dans le troisième chapitre, l'auteur identifie une nouvelle tendance dans la conception de la divinité dont Hermarque et Philodème, mais aussi Antiphane, semblent se faire les porte-parole. Cette tendance consiste à attribuer à la divinité des activités comme la respiration, le sommeil, l'alimentation, mais aussi la conversation et le sexe. Or, cette divergence par rapport à la doctrine du maître n'est pas considérée par l'auteur comme une forme d'hétérodoxie ou de dissidence (deux concepts dont la légitimité est, d'ailleurs, très discutée par les savants). En effet, la possibilité que le dieu soit ou ne soit pas capable d'agir n'est pas une question sur laquelle, selon les épicuriens, on puisse avoir une véritable connaissance. Tout ce que la prolepse nous fournit est l'idée du dieu comme doué d'incorruptibilité et de béatitude, éléments qui ne sont pas plus mis en discussion par les épicuriens postérieurs que par Épicure lui-même. Au-delà de ces données, on ne peut qu'émettre sur le dieu des hypothèses, lesquelles constituent, comme le dit l'auteur, un passage «dalla prolessi all'occulto » (p. 71). La divergence des épicuriens postérieurs ne serait donc que l'exploration d'une conception alternative à celle du maître, mais toujours cohérente avec les données de la prolepse.

À la lumière de cette approche, les chapitres trois et quatre ont le mérite de souligner l'originalité et les caractères propres de la réflexion théologique de chacun des épicuriens en présentant l'épicurisme comme un courant philosophique dynamique et en évitant de ramener toute forme d'originalité à une tentative de dissidence. Dans ces deux chapitres, outre l'examen des interprétations d'Hermarque, Antiphane et Philodème, l'auteur se confronte au principe d'isonomie selon Métrodore comme élément qui pourrait expliquer l'incorruptibilité des dieux, à la question des effets des émanations divines sur le bien-être des individus selon Polyène, aux raisons de la critique du mythe d'Er par Colotès, aux doutes de Pythoclès et Léonteus sur la possibilité de connaître dieu, au trilemme sans solution de Polystrate, à la question des $\mu \varepsilon \tau \alpha \kappa o ́ \sigma \mu 1 \alpha /$ intermundia chez

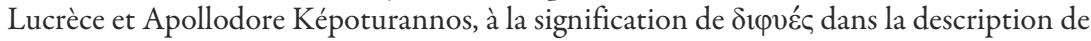
la double nature divine chez Démétrius Lacon, à la théorie de Nicasicratès transmise par Philodème sur les pensées éternelles des dieux, à la signification de la Vénus lucrétienne, à la célèbre théorie du quasi sanguis transmise par Cicéron, à la rêverie utopiste de Diogène d'OEnoanda et enfin à la critique de la divination et du destin de Boéthos et de Diogénien.

Le cinquième et dernier chapitre porte sur les implications éthiques des théologies de Démocrite et d'Épicure. L'objectif visé par l'auteur est de prouver que, malgré le caractère mortel de la divinité démocritéenne, l'inactivité de la divinité épicurienne, et l'activité partielle mais autocentrée de la divinité des épicuriens postérieurs, les théologies atomistes peuvent jouer un rôle important du point de vue de l'éthique et de la morale. Les cibles critiques sont évidemment tous ceux qui, de l'Antiquité jusqu'à nos jours, ont accusé les épicuriens d'être des athées camouflés. Cette accusation se fonderait, selon l'auteur, sur une conception de la divinité dérivée de la tradition des religions révélées : la conception d'une divinité bienveillante et à l'écoute, capable d'entrer en relation avec l'humanité et de l'inspirer directement. Toutefois, l'auteur pousse son analyse plus loin et, de la constitution d'un juste regard historique sur les théologies atomistes, passe à l'examen des idées préconçues qui font obstacle à la compréhension de l'efficacité éthique de ce genre de théologie. Autrement dit, l'auteur ne se pose pas seulement la question de 
savoir comment l'existence d'un dieu qui ne nous écoute pas peut avoir un effet positif sur l'être humain et sur sa conduite, mais aussi pourquoi il est si difficile, pour nous aujourd'hui, de répondre à cette question. Avec un geste dont la portée philosophique se révèle très intéressante, la réflexion sur la légitimité de l'emploi de catégories comme celles de « théologie », de « prière » et même de « dieu », dans le cadre des conceptions atomistes de la divinité, devient un miroir pour le lecteur qui se voit invité à réfléchir sur ces catégories mêmes et sur sa façon de les entendre. Le résultat de cette cinquième partie est la reconstitution de l'aspect le plus concret des théologies atomistes : une conception différente, nouvelle et «inattuale » de la relation à la divinité, dont la perfection et la béatitude apportent des bénéfices indirects à l'être humain : ce dernier s'en inspire et le

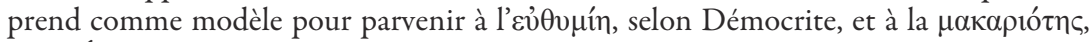
selon Épicure et ses disciples.

Cet ouvrage, riche et intéressant, parvient aux objectifs fixés dans son introduction. La cohérence et la solidité des différentes doctrines reconstituées démontrent toute la légitimité qu'il y a à considérer les réflexions atomistes sur le divin comme de véritables théologies, caractérisées par une remarquable portée éthique, et dont il est possible de reconstituer l'histoire. Cette histoire toutefois ne peut être reconstituée, nous rappelle l'auteur, qu'à condition de respecter les caractères particuliers des réflexions de chacun de ses protagonistes.

Simone Vezzoli, Arcesilao di Pitane. L'origine del platonismo neoaccademico, Turnhout, Brepols, 2016 (Philosophie hellénistique et romaine), 300 p., ISBN 978-2-503-55029-9.

Les travaux sur la Nouvelle Académie n'ont pas manqué durant les trente dernières années, mais très rares sont les monographies entièrement consacrées à l'un de ses trois grands représentants, l'exception qui confirme la règle étant l'ouvrage de Charles Brittain sur Philon de Larissa (Oxford, 2001). L'ouvrage de Simone Vezzoli (désormais S. V.) sur Arcésilas est issu d'une thèse de doctorat soutenue à Macerata en 2010 et a inauguré une nouvelle collection des éditions Brepols, Philosophie hellénistique et romaine (HФR), dirigée par C. Lévy et G. Reydams-Schils et qui compte trois ans plus tard déjà huit titres parus.

L'ouvrage de S. V. sur Arcésilas comprend une introduction, deux parties, Analisi (134 pages) et Fonti (122 pages), suivies par une bibliographie. Il ne comporte aucun index des passages, noms ou notions cités, ce qui est regrettable. La seconde partie recense l'ensemble des témoignages mentionnant Arcésilas; ils sont cités en grec ou latin, traduits en italien et assortis à chaque fois d'indications sur leur contexte, souvent d'un bref apparat critique et de renvois à d'autres témoignages parallèles ou à la littérature secondaire, voire d'une discussion détaillée dans deux ou trois cas controversés (par exemple le fameux passage de Plutarque, $A d v$. Col. 26-29, discuté p. 193-195). S. V. a réuni 161 témoignages et 10 dubia, mais plusieurs figurent sous deux numéros différents (mais ne sont cités qu'une seule fois par S. V.) s'ils nous ont été transmis par un auteur qui en cite un autre. Par exemple, les passages des Silles de Timon d'Athènes sur Arcésilas sont référencés d'abord au tout début des Fonti (F 2a à F 7), mais ne sont cités que dans les témoignages F 107, 115, 116 et 121, à savoir les passages de Diogène Läerce et Eusèbe (citant Numénius) qui citent les Silles. Les témoignages sont en effet présentés par S. V. dans l'ordre chronologique des sources. Cela permet de se faire une idée de la stratification 